\section{Indicadores dos atributos físicos e sociais da vizinhança obtidos pelo método de Observação Social Sistemática}

\author{
Indicators of physical and social neighborhood \\ attributes measured by the Systematic Social \\ Observation method
}

\section{Indicadores sobre el ambiente físico y social de un vecindario obtenidos por el método de la Observación Social Sistemática}

\section{Resumo}

O local de moradia é fortemente modelado pela posição social, indicando que características da vizinhança podem ser importantes contribuintes para as iniquidades em saúde. O objetivo foi construir indicadores do ambiente físico e social em um contexto urbano a partir das variáveis obtidas pelo método de Observação Social Sistemática (OSS) e analisá-los de acordo com o Índice de Vulnerabilidade à Saúde (IVS). O instrumento foi desenvolvido com o objetivo de conhecer as características do entorno físico e social do local de moradia de residentes de dois distritos sanitários de Belo Horizonte, Minas Gerais, Brasil. Os dados foram coletados entre os meses de abril e junho de 2011. Na construção dos indicadores simples, foram calculadas razões do número de itens observados por residência para cada segmento. Na construção dos indicadores compostos, foi utilizado o método de análise de componentes principais via matriz de covariâncias. A amostra final foi composta por 1.295 segmentos de ruas aninhados em 147 vizinhanças. Percebemos que os indicadores referentes às condições das ruas e itens de trânsito, mobilidade, estético, caracterização dos imóveis, desordem física, segurança e serviços apresentaram um comportamento dose-resposta em relação ao IVS (valor de $p<0,05$ ). Os indicadores referentes ao local para prática de atividade física e lazer não apresentaram diferenças significativas. Os indicadores demonstraram comportamento coerente diante de diferentes estratos do indice de vulnerabilidade da saúde e mostraram-se adequados dentro de cada domínio e subdomínio criados.

Distribuição Espacial da População; Indicadores Sociais; Saúde Urbana

\author{
Dário Alves da Silva Costa 1,2 \\ Sueli Aparecida Mingoti 1,3 \\ Amanda Cristina de Souza Andrade 1 \\ César Coelho Xavier 1,4 \\ Fernando Augusto Proietti 1,5 \\ Waleska Teixeira Caiaffa 1,2
}

doi: 10.1590/0102-311X00026316

\author{
Correspondência \\ D. A. S. Costa \\ Observatório de Saúde Urbana de Belo Horizonte, Universidade \\ Federal de Minas Gerais. \\ Av. Alfredo Balena 190, Belo Horizonte, MG 30130-100, Brasil. \\ darioalves_sc@yahoo.com.br \\ 1 Observatório de Saúde Urbana de Belo Horizonte, \\ Universidade Federal de Minas Gerais, Belo Horizonte, Brasil. \\ 2 Faculdade de Medicina, Universidade Federal de Minas \\ Gerais, Belo Horizonte, Brasil. \\ 3 Instituto de Ciências Exatas, Universidade Federal de Minas \\ Gerais, Belo Horizonte, Brasil. \\ 4 Faculdade de Saúde e Ecologia Humana, Vespasiano, Brasil. \\ 5 Instituto René Rachou, Fundação Oswaldo Cruz, Belo \\ Horizonte, Brasil.
}




\section{Introdução}

Data de meados do século XIX a compreensão de trabalhadores, sindicalistas, políticos e médicos de que a saúde resulta das condições de vida e do contexto em que as pessoas vivem, contribuindo para o entendimento do processo saúde-doença ${ }^{1}$. As estratégias, então centradas na melhoria das condições de vida das famílias e dos bairros, incluíram a participação política dos envolvidos no processo, que passou a ser fundamental 2. Nessa linha, nas últimas décadas, as características do entorno físico e social do local de moradia tornaram-se cada vez mais presentes na pesquisa epidemiológica, ampliando a compreensão da ocorrência dos eventos relacionados à saúde nas populações 3 .

Nos últimos anos, tem aumentado o interesse no território frente à visibilidade dos efeitos da vizinhança na saúde de seus moradores 4 . Estudos sobre efeitos da vizinhança também têm sido potencializados, devido ao grande interesse no entendimento das causas das desigualdades sociais em saúde ${ }^{3}$. O local de moradia é fortemente modelado pela posição social, indicando que características da vizinhança podem ser importantes contribuintes para as iniquidades em saúde, conceituadas como as desigualdades injustas 5 .

A incorporação de variáveis de contexto na saúde pública é uma importante contribuição de áreas como sociologia, educação e criminologia 6,7,8. Pode ser obtida a partir do relato da percepção dos sujeitos amostrados em uma investigação e por meio da observação direta de elementos do contexto 9 .

Estudos internacionais utilizando informação de contexto obtida de forma subjetiva investigam desde eventos em saúde, como doenças cardiovasculares, a hábitos e comportamentos associados às causas desses eventos 10,11,12. No Brasil, podemos citar alguns, dentre vários estudos: o Inquérito de Saúde dos Adultos, na Região Metropolitana de Belo Horizonte (Minas Gerais), que avaliou a percepção do entorno social da vizinhança e morbidade autorreferida 13, e o Estudo Saúde em Beagá 14, que investigou as qualidades psicométricas de características contextuais medidas a partir da percepção do ambiente social e físico de residentes participantes do inquérito, bem como a associação com a atividade física em diferentes níveis socioeconômicos 15 .

De forma objetiva, os atributos físicos e sociais da vizinhança podem ser mensurados pelo método da Observação Social Sistemática (OSS). A OSS é definida como a observação direta das condições físicas e interações sociais que ocorrem no local de moradia ou vizinhança 16 , obtida de acordo com protocolos, procedimentos e regras explícitas que permitam a replicação e o uso da lógica da inferência científica 6 .

Representa importante ferramenta complementar em estudos epidemiológicos, sendo capaz de capturar, de forma válida, confiável e independente, características físicas e sociais da vizinhança, consideradas variáveis integrais e impossíveis de serem acessadas por meio de inquéritos populacionais tradicionais.

Cada vez mais utilizada em saúde pública, a OSS possibilita investigar a associação entre atributos físicos e sociais da vizinhança e diversos eventos relacionados à saúde, tais como comportamentos e estilos de vida, podendo ser citados estudos sobre atividade física 17, deterioração física estrutural 18, autoavaliação da saúde 19 e urbanização 20.

Portanto, este artigo tem como objetivo construir indicadores do ambiente físico e social em um contexto urbano a partir das variáveis obtidas pelo método da OSS e analisá-los de acordo com um indicador territorial de vulnerabilidade à saúde.

\section{Métodos}

Esta investigação faz parte de um estudo epidemiológico multimétodos de saúde urbana, denominado Estudo Saúde em Beagá 21, cujo referencial teórico 22 considera que características sociais e físicas definem o contexto urbano e são moduladas por fatores proximais e distais e operam em múltiplos níveis. Foi desenvolvido pelo Observatório de Saúde Urbana de Belo Horizonte (OSUBH), da Faculdade de Medicina, Universidade Federal de Minas Gerais (UFMG) - para caracterizar estilos e qualidade de vida da população de Belo Horizonte, viabilizando estratégias de prevenção e promoção de hábitos de vida saudáveis. Posteriormente, realizou-se o Projeto Observação Social Sistemática, que teve como objetivos conhecer as características do entorno físico e social do local de moradia dos residentes 
urbanos participantes do inquérito e determinar e quantificar a correlação entre tais características e a ocorrência de eventos relacionados a saúde 23 .

\section{Estudo Saúde em Beagá}

Inquérito amostral domiciliar de base populacional estratificado e em conglomerados em três estágios (setor censitário; domicílio; indivíduo), realizado em 2008-2009, em 2 dos 9 Distritos Sanitários (DS) de Belo Horizonte: Oeste e Barreiro, correspondentes a cerca de 20\% da população da cidade 24 e escolhidos devido à proximidade geográfica e heterogeneidade interna de indicadores demográficos, socioeconômicos e de saúde 22,25. Em cada domicílio amostrado, um adulto foi aleatoriamente selecionado para a entrevista, após consentimento livre e esclarecido, aprovado pelo Comitê de Ética em Pesquisa da UFMG, sob parecer no 253/06. Informações mais detalhadas sobre esse inquérito podem ser obtidas em estudos anteriores 21,25 .

\section{Projeto Observação Social Sistemática}

Intitulado Observação Social Sistemática: Aplicação da Observação Direta das Condições Físicas e Sociais de uma Vizinhança, esse estudo propôs a utilização da OSS 23,26 para aferição das características físicas e sociais das vizinhanças, tendo as qualidades de confiabilidade avaliadas em estudo prévio 23 . O instrumento mostrou-se adequado para a observação de características com estabilidade temporal, principalmente quanto aos serviços, caracterização dos imóveis, ambiente para pedestres e segurança.

Construído a partir da literatura disponível na época e de várias "aproximações de campo" conduzidas previamente à investigação, foi delineado para capturar informações do entorno físico e social. O arsenal instrumental foi composto por instrumento de coleta, e o ferramental de apoio, por dois manuais 23 .

$\mathrm{O}$ instrumento de coleta, confeccionado com base na literatura e na experiência acumulada nas aproximações de campo, contém 215 questões em sua versão final, distribuídas em variáveis alocadas nos seguintes domínios: físico, social e atividade física, caracterização dos imóveis, da estética, dos serviços e da segurança. As questões apresentam escalas de medição variadas, com itens dicotômicos (p.ex.: presença ou ausência de passeios), itens de contagem (p.ex.: número de árvores no segmento), ordinais (p.ex.: qualidade da superfície das ruas) e contínuos (p.ex.: medições da largura do passeio).

Quanto ao ferramental de apoio ao instrumento de coleta, foram elaborados dois manuais: o primeiro, conceitual, contendo informações detalhadas sobre os procedimentos de campo; e o segundo, iconográfico, contendo fotos sobre questões geradoras de dúvidas, principalmente aquelas relacionadas à qualificação dos itens. O instrumento completo pode ser obtido diretamente com os autores.

\section{Desenho amostral}

A área de estudo correspondeu às unidades geográficas do inquérito de base populacional descrito anteriormente a partir da premissa de que indivíduos de um mesmo setor censitário pertencem a uma mesma vizinhança, mesmo embora a delimitação espacial desta última não coincida com o setor censitário 25,26,27. Os segmentos alcançados, mesmo que parcialmente por um entrevistado que, hipoteticamente, sai de sua residência e caminha 100 metros em qualquer direção, foram definidos como a área de alcance do entrevistado. Os segmentos alcançados por qualquer indivíduo do mesmo setor censitário foram definidos como a vizinhança do entrevistado.

A definição de vizinhança aqui adotada busca aproveitar a característica de aglomeração dos participantes no inquérito por setores censitários, consequente ao desenho amostral, e também considera a questão do deslocamento dos indivíduos no entorno das moradias. Dessa forma, 149 vizinhanças potenciais foram teoricamente definidas - mesmo número de setores censitários do estudo. Entretanto, dois setores censitários foram considerados como sendo uma mesma vizinhança por apresentarem domicílios sorteados em uma mesma rua e outros dois setores censitrários também foram agrupados considerando que no Censo Demográfico de 2000 era um único setor. Por meio da malha viária georreferenciada de Belo Horizonte, foram selecionados os segmentos de cada uma das 147 vizinhanças, resultando em uma base final de 2.975 segmentos com uma média de 20 segmentos por vizinhança. 
Após a definição dos segmentos que compunham cada vizinhança da base de amostra, foi definido um processo de amostragem sistemática dos segmentos em cada vizinhança. Os segmentos foram numerados em ordem sequencial de 1 (um) até o total de segmentos da vizinhança. A amostragem sistemática foi escolhida por distribuir melhor espacialmente os segmentos no setor e para evitar que vários segmentos de uma única rua fossem selecionados, o que poderia ocorrer no caso de uma amostra aleatória simples. A amostra final foi composta por 1.295 segmentos.

\section{Coleta dos dados}

Os dados foram coletados entre os meses de abril e junho de 2011. A unidade de estudo foi o segmento, exceto no domínio caracterização dos imóveis, no qual a unidade foi o imóvel. Para tanto, 50\% dos imóveis de cada segmento foram amostrados de forma sistemática.

Os segmentos foram observados por duplas, alteradas diariamente em sua composição de forma aleatória. Cada observador preencheu uma seção do instrumento, sendo permitida a comunicação entre eles.

Por se tratar de uma área heterogênea, com alguns setores em áreas de maior vulnerabilidade social, o trabalho de campo contou com o apoio dos gerentes dos distritos sanitários, bem como dos gerentes e dos agentes comunitários das unidades básicas de saúde dessas regiões.

\section{Análise dos dados}

\section{- Construção dos indicadores simples}

O primeiro passo foi eleger quais variáveis poderiam compor os indicadores. Para isso, foi realizada uma análise exploratória em que as variáveis foram selecionadas de acordo com a praticidade, a prevalência e o poder discriminatório das desigualdades espaciais. A seguir, as variáveis selecionadas foram reorganizadas de acordo com os seguintes domínios 28: D1: Condições das ruas e itens de trânsito; D2: Mobilidade; D3: Local para a prática de atividade física e lazer; D4: Caracterização dos imóveis; D5: Estético; D6: Desordem física; D7: Segurança; e D8: Serviços (Tabela 1).

Os indicadores simples, para as contagens, foram construídos a partir das razões do número de itens observados por residência para cada segmento (p.ex.: número de árvores por residência no segmento), de forma que tais indicadores pudessem ser entendidos como estimadores de razão 29, e os segmentos como conglomerados. Assim, as estimativas de médias e erros-padrão das vizinhanças foram obtidas pelo método de estimação por razão para conglomerados de tamanhos distintos.

Para o cálculo dos estimadores de razão por vizinhança e seus respectivos erros-padrão, foram utilizados modelos de regressão linear, considerando cada vizinhança como estrato, utilizando ainda como peso amostral o número de imóveis do segmento.

Para variáveis categóricas, ou seja, que representavam a presença de certas características (p.ex.: pavimentação do segmento), os indicadores simples foram construídos por meio do método de estimadores de razão obtidos pela proporção média de segmentos que apresentam tal característica por vizinhança.

Foram construídos indicadores simples para as 147 vizinhanças, que representavam as estimativas médias das razões observadas.

\section{- Construção dos indicadores compostos}

Para cada domínio, foi comstruído um indicador composto, a partir dos indicadores simples correspondentes. Alguns itens foram invertidos para manter a lógica dos indicadores dentro do mesmo domínio, de forma que apresentassem a mesma direção. A fim de permitir a comparação e a agregação de indicadores de diferentes medidas de escalas, foi realizada a transformação de todos os indicadores para valores entre 0 e 1 .

Foi utilizado o método de análise de componentes principais via matriz de covariâncias para identificar os indicadores compostos e estimar os respectivos escores ${ }^{30}$. Alguns domínios foram mais bem representados por dois ou três componentes. Nesses casos, foram criados subdomínios. O número 


\section{Tabela 1}

Itens utilizados na construção dos indicadores, conforme os domínios propostos.

\section{Domínio}

D1: Condições das ruas e itens de trânsito

D2: Mobilidade

D3: Local para a prática de atividade física e lazer

D4: Caracterização dos imóveis

D5: Estético

D6: Desordem física
Variáveis

1. Pavimentação do segmento

$$
\text { 2. Tipo (via) }
$$

3. Sinalização de transporte coletivo *

4. Condição de pavimentação (mato, buraco, saliência)

5. Sentido de circulação (sentido duplo)

6. Número de faixas (até duas faixas)

7. Sinalização de estacionamento proibido *

8. Sinalização exclusiva para deficientes/idosos

9. Itens de trânsito (canteiro, lombada, radar, semáforo) *

10. Observação de infrações

11. Fluxo de veículos

1. Pavimentação do passeio *

2. Rampa de acesso ou piso tátil *

3. Escadaria ou corrimão

4. Árvores que produzam sombra *

5. Largura do passeio na menor extremidade (metros) *

6. Itens de trânsito para pedestres (grade, faixa, passarela) *

7. Condição de pavimentação (mato, buraco, saliência)

8. Obstrução (banca, lixeiras, árvores, postes, outros)

9. Percepção do ambiente agradável para deslocamento *

1. Local para socialização (parques e praças) *

2. Local para prática de atividade física *

3. Percepção do ambiente como agradável pra atividade física *

1. Imóveis no segmento *

2. Imóveis com até dois pavimentos *

3. Imóveis comerciais *

4. Imóveis em construção/reforma *

5. Propaganda de venda de imóveis

1. Propaganda de eventos políticos/partidários *

2. Propaganda de comércio de tabaco, álcool, fast food

$$
\text { 3. Grafite }
$$

4. Jardim/árvore *

5. Espaço aberto

6. Ambiente limpo *

7. Barulho

8. Música *

1. Imóveis pichados

2. Imóveis com sinais de deterioração 3. Lixo (agulha, cigarro, lata, preservativos) *

4. Equipamentos públicos pichados *

5. Pichação e/ou deterioração de imóveis

(continua) 
Tabela 1 (continuação)

Domínio

D7: Segurança

D8: Serviços

* Item utilizado nos indicadores simples e compostos.

\section{Variáveis}

1. lluminação pública *

2. Aviso de propriedade protegida por cão

3. Aviso de propriedade protegida por alarme

4. Propriedade protegida por arame

5. Propriedade protegida por portão/muro pontiagudo

6. Propriedade protegida por janela com grade

7. Propriedade protegida por cerca elétrica

8. Propriedade com porteiro

9. Propriedade protegida por caco de vidro

10. Propriedade protegida por câmera

11. Itens de segurança *

12. Policiamento *

1. Coletor de lixo público ou privado *

2. Lixeira

3. Telefone público

4. Ensino infantil

5. Ensino Fundamental e Médio *

6. Ensino Superior

7. Local de compra de alimentos para serem preparados *

\section{Mercearia}

9. Hipermercado/supermercado

10. Local para lanches rápidos *

11. Padaria

12. Restaurante

13. Frigorífico

14. Local para compra de verduras *

15. Loja de vestuário e acessórios

16. Mecânica/acessórios automotivos *

17. Banca de jornal

18. Drogaria

19. Bares

20. Igreja

21. Academia pública ou privada *

22. Salão de beleza

23. Saúde pública

24. Saúde privada *

de componentes foi definido com base nas porcentagens de variância total explicada. Além disso, foi verificado o alfa de Cronbach ${ }^{31}$ para avaliar a consistência do conjunto final de variáveis que permaneceram em cada domínio. Para melhor interpretação dos indicadores compostos estimados, todos foram transformados em uma escala de 0 a 5.

Os indicadores construídos foram então avaliados de acordo com o Índice de Vulnerabilidade à Saúde (IVS). O IVS, um proxy de indicador social, é um indicador composto, ponderado, criado pelo município, utilizando características socioeconômicas e de saneamento obtidas a partir das informações do censo brasileiro de 2010 32, cuja unidade geográfica é o setor censitário. O IVS é categorizado em baixo, médio, elevado e muito elevado, conforme pontos de corte definidos a partir do desvio padrão em torno da média. Quanto maior a categoria do IVS, maior a vulnerabilidade da vizinhança 33 .

A escolha do IVS se deu pelo fato de ser um indicador simples e de fácil compreensão, importante para o planejamento e a avaliação das políticas de saúde, mostrando-se capaz de discriminar as desigualdades do adoecer e morrer no território, quando conjugado a outras estratégias. 
As análises foram realizadas no software Stata versão 12.1 (StataCorp LP, College Station, Estados Unidos) e apresentadas de acordo com os estratos de IVS. Em todas elas foi considerado o peso amostral de acordo com o plano amostral e coleta de dados. O teste não paramétrico de Kruskal-Wallis 34 foi utilizado nas comparações entre os estratos de IVS. Foi adotado o nível de significância de $5 \%$.

\section{Resultados}

A amostra final foi composta por 1.295 segmentos de ruas aninhados em 147 vizinhanças. O comprimento médio dos segmentos foi de 122,2 metros ( $\pm 44,6$ ); cada vizinhança possuía, em média, 8,8 segmentos $( \pm 2,0)$. Em relação à distribuição do IVS nas 147 vizinhanças, observou-se percentual de $22,5 \%$ na categoria de risco baixo, $38,1 \%$ no médio, $31,2 \%$ no elevado e $8,2 \%$ no muito elevado.

De acordo com os oito domínios criados, as variáveis observadas em cada segmento assim se agruparam: D1: Condições das ruas e itens de trânsito (11 variáveis); D2: Mobilidade (9 variáveis); D3: Local para a prática de atividade física e lazer (3 variáveis); D4: Caracterização dos imóveis (5 variáveis); D5: Estético (8 variáveis); D6: Desordem física (5 variáveis); D7: Segurança (12 variáveis); e D8: Serviços (24 variáveis).

\section{Indicadores simples}

Na Tabela 2 são apresentados os indicadores simples, construídos, considerando a distribuição dos itens avaliados pela densidade de residências. No domínio D1, o número de segmentos do tipo via, sinalização de estacionamento proibido, itens de trânsito, veículos cometendo infrações e fluxo de veículos, apresentaram uma redução quando observamos do IVS baixo para o muito elevado, sendo as diferenças significativas a $1 \%$. Os indicadores que não apresentaram diferenças significativas foram homogêneos em todas as categorias de IVS.

Na avaliação do domínio D2, observamos uma redução na distribuição dos indicadores à medida que aumenta o risco do IVS. Essa redução pode ser observada, como exemplo, no número de árvores que produzem sombra. Enquanto no IVS baixo a densidade era de 734,2 árvores/1.000 residências, no IVS muito elevado foi de apenas 64,5 árvores $/ 1.000$ residências $(\mathrm{p}<0,001)$. Para o indicador que marcava a presença de escadaria e corrimão, não foram encontradas diferenças significativas, sendo notadamente baixa a presença em todas as categorias de IVS. Nenhum dos indicadores do domínio D3 apresentou diferenças significativas, provavelmente pela baixa frequência e poucas opções de itens para compor esse domínio.

No domínio D4, os indicadores mostraram-se heterogêneos entre as diferentes categorias de IVS. Porém, foi observada uma redução gradativa apenas nos indicadores referentes à presença de imóveis em construção/reforma $(p=0,013)$ e propaganda de venda de imóveis $(p<0,001)$.

No domínio D5 foi observado um comportamento bem definido entre os indicadores. Propaganda de eventos políticos, grafite, espaços aberto e som de música aumentam à medida que aumenta o risco do IVS. Já indicadores que marcam a presença de jardins/árvores, ambiente limpo e fontes de barulho reduzem à medida que aumenta o risco do IVS. Apenas a presença de propaganda de comércio (tabaco, álcool e fast food) não apresentou diferença significativa entre os estratos de IVS. A presença de lixo e de equipamentos públicos pichados também apresentaram diferenças significativas quando comparados o IVS baixo com o elevado.

A presença de pichação e/ou deterioração de imóveis foi o único indicador do domínio D6 a apresentar gradiente dose-resposta. Em vizinhanças com IVS baixo, existiam aproximadamente 628,9 imóveis pichados e/ou deteriorados a cada 1.000 residências. Essa medida cresce com o aumento do risco do IVS, sendo que, em vizinhanças com IVS muito elevado, temos aproximadamente 811,3 imóveis pichados e/ou deteriorados a cada 1.000 residências $(\mathrm{p}=0,026)$. Na avaliação de indicadores de segurança (domínio D7), vizinhanças com IVS baixo apresentaram maior presença desses itens, diminuindo à medida que aumentava o IVS. Considerando a combinação de todos os itens de segurança nos imóveis, observamos que vizinhanças com IVS baixo têm aproximadamente média de 109,4 itens de segurança a cada 1.000 residências. Esse valor se reduz à medida que se elevam as categorias 
Tabela 2

Indicadores simples por 1.000 residências distribuídos por categoria do Índice de Vulnerabilidade da Saúde (IVS). Belo Horizonte,

Minas Gerais, Brasil, 2011.

\begin{tabular}{|c|c|c|c|c|c|}
\hline \multirow[t]{3}{*}{ Domínios } & \multicolumn{4}{|c|}{ IVS } & \multirow[t]{3}{*}{ Valor de $p$} \\
\hline & Baixo $(n=33)$ & Médio ( $n=56$ ) & Elevado $(n=46)$ & Muito elevado ( $n=12)$ & \\
\hline & Mediana (IQ) & Mediana (IQ) & Mediana (IQ) & Mediana (IQ) & \\
\hline \multicolumn{6}{|l|}{ D1: Condições das ruas e itens de trânsito } \\
\hline Pavimentação do segmento * & $100,0(0,0)$ & $100,0(0,0)$ & $100,0(0,0)$ & $100,0(0,0)$ & 0,873 \\
\hline Tipo (via) * & $100,0(0,0)$ & $100,0(0,0)$ & $100,0(16,5)$ & $76,1(29,1)$ & $<0,001 * *$ \\
\hline Sinalização de transporte coletivo & $19,6(29,8)$ & $21,8(17,6)$ & $9,9(11)$ & $15,2(20,6)$ & $<0,001 * *$ \\
\hline $\begin{array}{l}\text { Condição pavimentação (mato, } \\
\text { buraco, saliência) }\end{array}$ & $729,4(328)$ & $376,9(120,4)$ & $366(181,1)$ & $385,2(319,7)$ & $<0,001 * *$ \\
\hline Sentido circulação (sentido duplo) * & $75,4(37,6)$ & $95,8(16,1)$ & $100,0(13,0)$ & $100,0(11,2)$ & $<0,001$ ** \\
\hline Número de faixas (até duas faixas) * & $100,0(0,0)$ & $100,0(2,3)$ & $100,0(0,0)$ & $100,0(0,0)$ & 0,388 \\
\hline Sinalização estacionamento proibido & $47,6(65,5)$ & $11,3(27,2)$ & $1,4(12,7)$ & $0,0(13,7)$ & $<0,001 * *$ \\
\hline Sinalização exclusiva deficientes/idosos & $0,0(0,0)$ & $0,0(0,0)$ & $0,0(0,0)$ & $0,0(0,0)$ & 0,865 \\
\hline $\begin{array}{l}\text { Itens de trânsito (canteiro, lombada, } \\
\text { radar, semáforo) }\end{array}$ & $149,7(133,8)$ & $86,8(86,1)$ & $47,5(61,7)$ & $31,2(25,9)$ & $<0,001 * *$ \\
\hline Observação de infrações & $28,2(50,0)$ & $9,9(15,1)$ & $7,8(18,2)$ & $0,0(6,8)$ & $<0,001 * *$ \\
\hline Fluxo de veículos *** & $117,3(142,6)$ & $44,5(48,5)$ & $25,9(28,9)$ & $28,0(39,3)$ & $<0,001 * *$ \\
\hline \multicolumn{6}{|l|}{ D2: Mobilidade } \\
\hline Pavimentação do passeio * & $100,0(0,0)$ & $100,0(7,0)$ & $96,1(16,5)$ & $77,5(28,1)$ & $<0,001 * *$ \\
\hline Rampa de acesso ou piso tátil & $36,4(69,7)$ & $10,6(30,6)$ & $0,0(9,8)$ & $0,0(5,1)$ & $<0,001 * *$ \\
\hline Escadaria ou corrimão & $8,1(22,2)$ & $0,0(5,8)$ & $0,0(14,1)$ & $0,0(11,7)$ & 0,124 \\
\hline Árvores que produzam sombra & $734,2(488,3)$ & $291,2(243,9)$ & $169,3(260,7)$ & $64,5(92,7)$ & $<0,001 * *$ \\
\hline $\begin{array}{l}\text { Largura do passeio na menor extremidade } \\
\text { (metros) }\end{array}$ & $1,7(0,5)$ & $1,8(0,5)$ & $1,5(0,7)$ & $0,9(0,6)$ & $<0,001 * *$ \\
\hline $\begin{array}{l}\text { Itens trânsito para pedestres (grade, faixa, } \\
\text { passarela) }\end{array}$ & $22,7(33,3)$ & $6,3(16,4)$ & $0,0(6,2)$ & $0,0(5,1)$ & $<0,001 * *$ \\
\hline 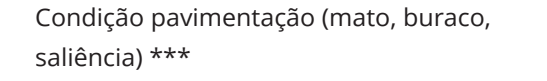 & $214,3(109,3)$ & $135,3(43,0)$ & $105,6(72,0)$ & $93,2(66,5)$ & $<0,001 * \star$ \\
\hline $\begin{array}{l}\text { Obstrução (banca, lixeiras, árvores, } \\
\text { postes, outros) }\end{array}$ & $243,9(170,7)$ & $159,5(91,3)$ & $151,0(87,5)$ & $151,1(65,9)$ & 0,002 ** \\
\hline $\begin{array}{l}\text { Percepção do ambiente agradável para } \\
\text { deslocar * }\end{array}$ & $67,5(45,3)$ & $53,7(42,5)$ & $30,4(36,9)$ & $13,8(36,0)$ & $<0,001 * *$ \\
\hline \multicolumn{6}{|l|}{$\begin{array}{l}\text { D3: Local para a prática de atividade } \\
\text { física e lazer }\end{array}$} \\
\hline Local para socialização (parques e praças) & $0,0(8,1)$ & $0,0(0,0)$ & $0,0(6,6)$ & $0,0(6,9)$ & 0,645 \\
\hline Local para prática de atividade física & $0,0(10,3)$ & $0,0(7,8)$ & $0,0(7,5)$ & $3,3(13,9)$ & 0,787 \\
\hline $\begin{array}{l}\text { Percepção do ambiente como agradável } \\
\text { para atividade física * }\end{array}$ & $2,5(17,0)$ & $1,9(13,7)$ & $0,0(8,6)$ & $1,8(10,7)$ & 0,429 \\
\hline \multicolumn{6}{|l|}{ D4: Caracterização dos imóveis } \\
\hline Imóveis no segmento *** & $132,5(35,6)$ & $119,3(15,1)$ & $113,9(15,8)$ & $117,4(19,3)$ & $<0,001 * *$ \\
\hline Imóveis com até dois pavimentos *** & $75,8(65,3)$ & $102,8(13,0)$ & $98,0(90,2)$ & $97,2(10,5)$ & $0,001 * *$ \\
\hline Imóveis comerciais & $264,5(210,3)$ & $259,6(121,4)$ & $145,4(98,3)$ & $176,1(56,6)$ & $<0,001 * *$ \\
\hline Imóveis em construção/reforma & $41,7(38,2)$ & $17,2(35,5)$ & $17,0(25,3)$ & $13,9(67,5)$ & 0,013 ** \\
\hline Propaganda de venda de imóveis * & $50,7(39,4)$ & $24,5(27,2)$ & $17,1(23,0)$ & $12,7(26,4)$ & $<0,001 * *$ \\
\hline
\end{tabular}

(continua) 
Tabela 2 (continuação)

\begin{tabular}{|c|c|c|c|c|c|}
\hline \multirow[t]{3}{*}{ Domínios } & \multicolumn{4}{|c|}{ IVS } & \multirow[t]{3}{*}{ Valor de $p$} \\
\hline & & & & Muito elevado $(n=12)$ & \\
\hline & Mediana (IQ) & Mediana (IQ) & Mediana (IQ) & Mediana (IQ) & \\
\hline \multicolumn{6}{|l|}{ D5: Estético } \\
\hline Propaganda de eventos políticos/partidários * & $17,9(32,5)$ & $28,2(44,9)$ & $45,4(47,7)$ & $71,9(111,5)$ & $<0,001 * *$ \\
\hline $\begin{array}{l}\text { Propaganda de comércio de tabaco, álcool, } \\
\text { fast food * }\end{array}$ & $9,5(22,1)$ & $7,1(14,5)$ & $8,2(12,3)$ & $6,8(13,0)$ & 0,836 \\
\hline Grafite & $16,5(24,4)$ & $23,9(43,5)$ & $49,3(60,1)$ & $33,3(21,1)$ & $0,002 * \star$ \\
\hline Jardim/Árvore & $674,7(341,6)$ & $419,5(157,2)$ & $354,5(214,7)$ & $304,1(216,8)$ & $<0,001 * *$ \\
\hline Espaço aberto * & $41,7(45,9)$ & $79,3(30,2)$ & $83,1(31,8)$ & $90,4(33,0)$ & $<0,001 * *$ \\
\hline Ambiente limpo * & $62(31,8)$ & $46,0(32,8)$ & $29,8(33,2)$ & $8,8(9,1)$ & $<0,001 * *$ \\
\hline Barulho * & $97,8(17,4)$ & $88,4(24,0)$ & $82,9(30,3)$ & $60,2(28,1)$ & $0,007 * \star$ \\
\hline Música * & $0,0(12,5)$ & $10,2(24,5)$ & $19,5(28,1)$ & $23,6(41,4)$ & $<0,001 * *$ \\
\hline \multicolumn{6}{|l|}{ D6: Desordem física } \\
\hline Imóveis pichados & $381,0(161,8)$ & $466,1(237,6)$ & $343,3(198,2)$ & $400,4(287,3)$ & $0,013 * *$ \\
\hline Imóveis com sinais de deterioração & $433,8(359,2)$ & $406,5(208,5)$ & $553,1(239,7)$ & $650,9(227,7)$ & $<0,001 * *$ \\
\hline Lixo (agulha, cigarro, lata, preservativos) & $305,1(155,7)$ & $192,6(77,0)$ & $170,5(105,3)$ & $216,4(88,9)$ & $<0,001 * *$ \\
\hline Equipamentos públicos pichados & $217,7(213,6)$ & $191,9(121,3)$ & $149,9(93,6)$ & $179,4(103,5)$ & $0,011 * *$ \\
\hline Pichação e/ou deterioração de imóveis & $628,9(319,0)$ & $687,4(183,5)$ & $693,4(191,8)$ & $811,3(211,9)$ & $0,026 * *$ \\
\hline \multicolumn{6}{|l|}{ D7: Segurança } \\
\hline Iluminação pública & $552,9(281,9)$ & $345,8(129,6)$ & $289,6(108,2)$ & $326,5(64,6)$ & $<0,001 * *$ \\
\hline Aviso de propriedade protegida por cão & $0,0(16,7)$ & $0,0(7,6)$ & $0,0(7,2)$ & $0,0(5,1)$ & 0,565 \\
\hline Aviso de propriedade protegida por alarme & $333,3(344,3)$ & $57,9(78,0)$ & $16,0(54,1)$ & $0,0(6,8)$ & $<0,001 * *$ \\
\hline Propriedade protegida por arame & $83,3(99,9)$ & $37,3(58,0)$ & $27,6(79,5)$ & $34,0(91,9)$ & $0,001 * *$ \\
\hline $\begin{array}{l}\text { Propriedade protegida por portão/muro } \\
\text { pontiagudo }\end{array}$ & $325,2(199,8)$ & $130,5(98,7)$ & $69(61,1)$ & $43,5(94,9)$ & $<0,001 * *$ \\
\hline $\begin{array}{l}\text { Propriedade protegida por janela } \\
\text { com grade }\end{array}$ & $615,0(399,0)$ & $334(165,7)$ & $317,8(148,2)$ & $238,1(159,2)$ & $<0,001 * *$ \\
\hline Propriedade protegida por cerca elétrica & $508,3(242,8)$ & $124,2(154,1)$ & $12,2(65,6)$ & $0,0(26,5)$ & $<0,001 * *$ \\
\hline Propriedade com porteiro & $41,2(100,6)$ & $0,0(0,0)$ & $0,0(0,0)$ & $0,0(7,0)$ & $<0,001 * *$ \\
\hline Propriedade protegida por caco de vidro & $42,8(63,6)$ & $46,3(49,8)$ & $55,4(43,5)$ & $53,3(72,3)$ & 0,788 \\
\hline Propriedade protegida por câmera & $107,1(99,5)$ & $17,2(37,9)$ & $0,0(18,0)$ & $0,0(0,0)$ & $<0,001 * *$ \\
\hline Itens de segurança $* \star \star$ & $109,4(24,7)$ & $59,5(23,4)$ & $46,0(14,4)$ & $41,1(12,0)$ & $<0,001 * *$ \\
\hline Policiamento & $24,4(26,0)$ & $9,6(17,4)$ & $0,0(6,2)$ & $3,4(11,7)$ & $<0,001 * *$ \\
\hline
\end{tabular}

(continua)

de IVS; vizinhanças com IVS muito elevado apresentavam, em média, 41,1 itens de segurança a cada 1.000 residências $(\mathrm{p}<0,001)$.

O domínio D8 foi aquele com o maior número de variáveis avaliadas. Porém, muitos itens não apresentaram diferenças significativas, distribuindo-se de forma homogênea nas categorias de IVS. Para os indicadores que apresentaram diferenças significativas, percebemos uma maior presença nas vizinhanças de IVS baixo e uma redução em relação às outras categorias de IVS. Apenas a presença de igrejas apresentou comportamento inverso, com maior número nas vizinhanças de IVS muito elevado $(\mathrm{p}<0,001)$.

\section{Indicadores compostos}

Em relação aos indicadores compostos (Tabela 3), a análise de componentes principais resultou em um componente constituído de três itens para compor o domínio D1 com variância total explicada de 81,8\% e alfa de Cronbach de 0,775. Permaneceram "Sinalização de transporte coletivo", "Sinalização de estacionamento proibido" e "Itens de trânsito". Foi possível observar diferença significativa entre as categorias de IVS, sendo que, em vizinhanças com IVS baixo, o indicador apresentava mediana de 1,4 (IQ: 1,3$)$ e, em vizinhanças com IVS muito elevado, a mediana era de 0,1 (IQ: 0,3) (p < 0,001). 
Tabela 2 (continuação)

\begin{tabular}{|c|c|c|c|c|c|}
\hline \multirow[t]{3}{*}{ Domínios } & \multicolumn{4}{|c|}{ IVS } & \multirow[t]{3}{*}{ Valor de $p$} \\
\hline & Baixo $(n=33)$ & & Elevado $(n=46)$ & Muito elevado $(n=12)$ & \\
\hline & Mediana (IQ) & Mediana (IQ) & Mediana (IQ) & Mediana (IQ) & \\
\hline \multicolumn{6}{|l|}{ D8: Serviços } \\
\hline Coletor de lixo público ou privado & $244,9(247,9)$ & $255,5(122,6)$ & $229,5(245,8)$ & $75,3(77,0)$ & $0,001 * \star$ \\
\hline Lixeira & $10,2(53,3)$ & $5,6(20,7)$ & $0,0(8,1)$ & $0,0(0,0)$ & $0,004 * \star$ \\
\hline Telefone público & $25,0(32,8)$ & $23,5(30,6)$ & $12,8(19,7)$ & $8,5(16,7)$ & $<0,001 * *$ \\
\hline Ensino infantil & $0,0(0,0)$ & $0,0(8,4)$ & $0,0(5,5)$ & $0,0(12,6)$ & 0,357 \\
\hline Ensino Fundamental e Médio & $0,0(6,7)$ & $0,0(10,6)$ & $0,0(0,0)$ & $0,0(0,0)$ & 0,079 \\
\hline Ensino Superior & $0,0(0,0)$ & $0,0(0,0)$ & $0,0(0,0)$ & $0,0(0,0)$ & 0,912 \\
\hline $\begin{array}{l}\text { Local de compra de alimentos para serem } \\
\text { preparados }\end{array}$ & $13,3(27,2)$ & $11,9(17,3)$ & $9,4(17,5)$ & $17,9(25,3)$ & 0,528 \\
\hline Mercearia & $5,9(12,8)$ & $5,8(12,1)$ & $4,1(12,1)$ & $4,9(20,0)$ & 0,949 \\
\hline Hipermercado/Supermercado & $0,0(11,8)$ & $0,0(2,0)$ & $0,0(0,0)$ & $0,0(0,0)$ & 0,431 \\
\hline Local para lanches rápidos & $0,0(23,8)$ & $0,0(10,8)$ & $4,5(8,2)$ & $1,8(12,1)$ & 0,717 \\
\hline Padaria & $0,0(10,2)$ & $4,7(10,7)$ & $0,0(5,5)$ & $0,0(0,0)$ & $0,026 * *$ \\
\hline Restaurante & $0,0(12,7)$ & $0,0(9,2)$ & $0,0(4,7)$ & $0,0(0,0)$ & 0,411 \\
\hline Frigorífico & $0,0(0,0)$ & $0,0(2,0)$ & $0,0(4,1)$ & $0,0(5,1)$ & 0,751 \\
\hline Local para compra de verduras & $0,0(12,2)$ & $0,0(10,8)$ & $0,0(4,2)$ & $0,0(6,7)$ & 0,366 \\
\hline Loja de vestuário e acessórios & $20,4(93,3)$ & $6,3(16,8)$ & $5,7(11,0)$ & $0,0(13,3)$ & $0,006 * *$ \\
\hline Mecânica/Acessórios automotivos & $8,3(56,6)$ & $21,8(38,5)$ & $6,7(17,4)$ & $1,8(30,4)$ & $0,004 * *$ \\
\hline Banca de jornal & $0,0(6,8)$ & $0,0(0,0)$ & $0,0(0,0)$ & $0,0(0,0)$ & 0,259 \\
\hline Drogaria & $0,0(8,3)$ & $0,0(0,0)$ & $0,0(0,0)$ & $0,0(0,0)$ & 0,334 \\
\hline Bares & $20,6(42,0)$ & $18,6(24,0)$ & $21,4(17,5)$ & $27,2(36,0)$ & 0,989 \\
\hline Igreja & $0,0(9,5)$ & $11,6(16,3)$ & $13,3(14,0)$ & $19,7(10,7)$ & $<0,001 * *$ \\
\hline Academia pública ou privada & $0,0(13,3)$ & $0,0(0,0)$ & $0,0(0,0)$ & $0,0(0,0)$ & $0,034 * *$ \\
\hline Salão de beleza & $18,9(34,0)$ & $21,2(23,4)$ & $14,2(13,1)$ & $20,4(21,3)$ & 0,121 \\
\hline Saúde público & $0,0(0,0)$ & $0,0(0,0)$ & $0,0(0,0)$ & $0,0(0,0)$ & 0,999 \\
\hline Saúde privado & $0,0(20,4)$ & $0,0(0,0)$ & $0,0(0,0)$ & $0,0(0,0)$ & $0,040 * \star$ \\
\hline
\end{tabular}

IQ: distância interquartílica.

* Proporção de segmentos por setor;

* Teste de Kruskal-Wallis significativo a 5\%;

*** Indicador por 100 residências.

No domínio D2 foi necessário considerar dois subdomínios: o primeiro, representado pela "Pavimentação do passeio", "Árvores que produzam sombra”, "Largura do passeio de menor extremidade” e "Percepção do ambiente como agradável para deslocar"; e o segundo, composto por "Rampa de acesso ou piso tátil" e "Itens de trânsito para pedestres". Ambos os subdomínios apresentaram medianas significativamente maiores $(\mathrm{p}<0,01)$ nas vizinhanças com IVS baixo em comparação às vizinhanças com IVS muito elevado (Figura 1). O domínio apresentou um alfa de Cronbach de 0,722, e a variância total explicada pelos dois subdomínios conjuntamente foi de 71,4\%.

Diferentemente dos anteriores, o indicador do domínio D3 não apresentou heterogeneidade entre as categorias de IVS. A medida do alfa de Cronbach foi de apenas 0,591, e a variância explicada foi de 70,1\%. Com relação ao domínio D4, o alfa de Cronbach foi de 0,593, explicando 82,6\% da variância total. Foram novamente considerados dois subdomínios. O primeiro, de certa forma, representa a densidade de imóveis na vizinhança, composto por "Imóveis no segmento", "Imóveis com até dois pavimentos" e "Imóveis comerciais"; o segundo representa "Imóveis em construção/reforma”. Os dois apresentavam medianas maiores em vizinhanças com IVS baixo.

$\mathrm{Na}$ avaliação dos atributos estéticos das vizinhanças, o domínio D5 apresentou alfa de Cronbach de 0,558, e apenas 48,4\% da variância total foi explicada. Porém, foi possível observar diferenças quando comparado IVS baixo com muito elevado ( $<$ 0,001). Para os atributos "Propaganda de eventos políticos" e "Música", os valores tiveram a escala invertida para apresentarem a mesma direção dos atributos "Jardim/árvore" e "Ambiente limpo". 
Tabela 3

Indicadores compostos distribuídos por categoria do Índice de Vulnerabilidade da Saúde (IVS). Belo Horizonte, Minas Gerais, Brasil, 2011.

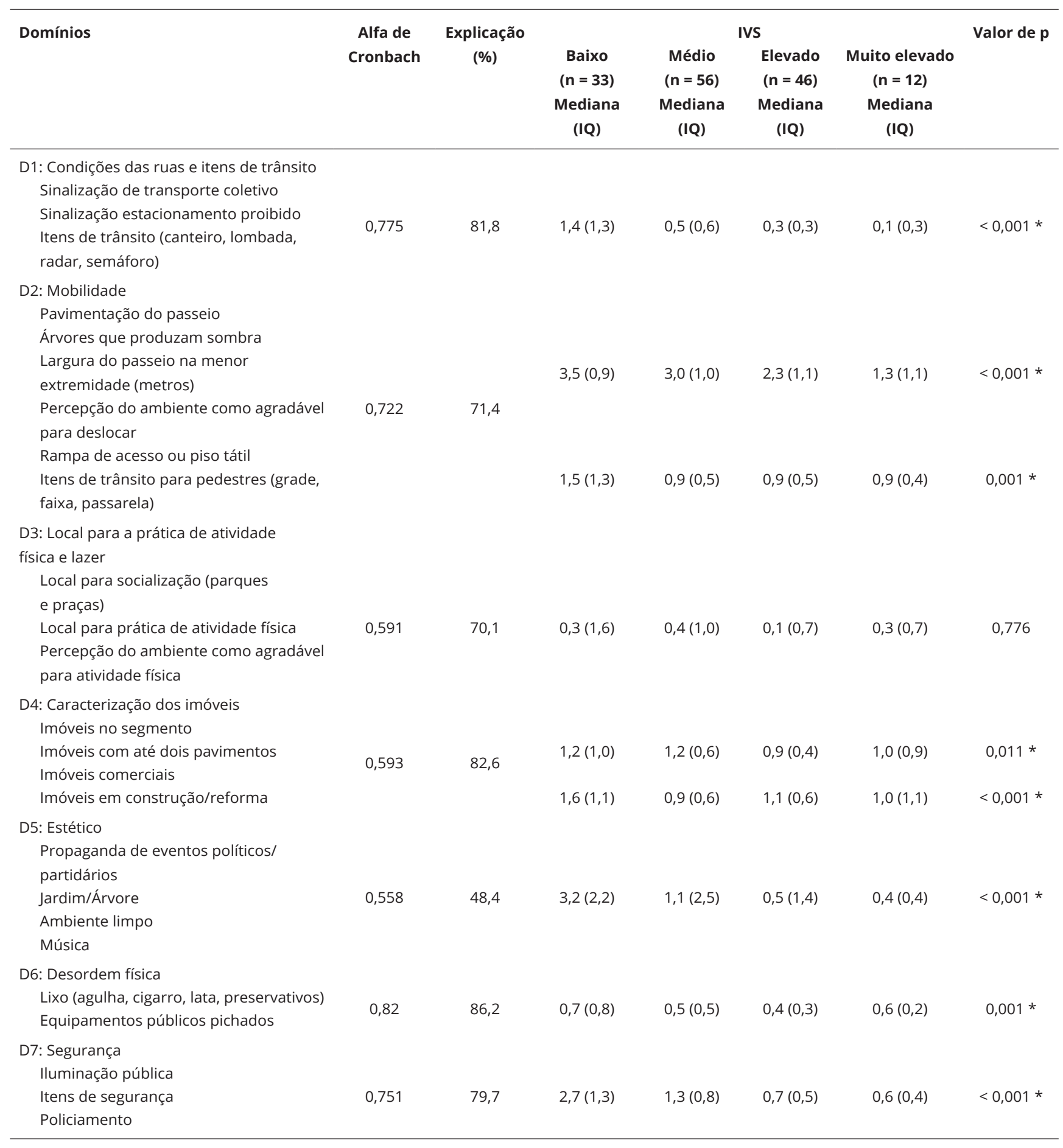

(continua) 
Tabela 3 (continuação)

\begin{tabular}{|c|c|c|c|c|c|c|c|}
\hline \multirow[t]{2}{*}{ Domínios } & \multirow{2}{*}{$\begin{array}{c}\text { Alfa de } \\
\text { Cronbach }\end{array}$} & \multirow{2}{*}{$\begin{array}{c}\text { Explicação } \\
\text { (\%) }\end{array}$} & \multicolumn{4}{|c|}{ IVS } & \multirow[t]{2}{*}{ Valor de $p$} \\
\hline & & & $\begin{array}{c}\text { Baixo } \\
(n=33) \\
\text { Mediana } \\
\text { (IQ) }\end{array}$ & $\begin{array}{c}\text { Médio } \\
(n=56) \\
\text { Mediana } \\
\text { (IQ) }\end{array}$ & $\begin{array}{c}\text { Elevado } \\
(n=46) \\
\text { Mediana } \\
\text { (IQ) }\end{array}$ & $\begin{array}{l}\text { Muito elevado } \\
\qquad(n=12) \\
\text { Mediana } \\
\text { (IQ) }\end{array}$ & \\
\hline \multicolumn{8}{|l|}{ D8: Serviços } \\
\hline \multicolumn{8}{|l|}{$\begin{array}{l}\text { Local de compra de alimentos para } \\
\text { serem preparados }\end{array}$} \\
\hline $\begin{array}{l}\text { Local para lanches rápidos } \\
\text { Local para compra de verduras }\end{array}$ & & & $0,7(0,8)$ & $0,5(0,2)$ & $0,5(0,2)$ & $0,6(0,1)$ & 0,003 * \\
\hline Saúde privado & 0,674 & 74,5 & & & & & \\
\hline \multicolumn{8}{|l|}{ Academia pública ou privada } \\
\hline $\begin{array}{l}\text { Coletor de lixo público ou privado } \\
\text { Ensino Fundamental e Médio }\end{array}$ & & & $1,2(1,3)$ & $1,4(0,5)$ & $1,0(1,0)$ & $0,4(0,3)$ & $<0,001$ * \\
\hline Mecânica/Acessórios automotivos & & & $2,1(0,9)$ & $2,3(0,5)$ & $2,1(0,3)$ & $2,2(0,1)$ & 0,023 * \\
\hline
\end{tabular}

IQ: distância interquartílica.

* Teste de Kruskal-Wallis significativo a 5\%.

Permaneceram no domínio D6 os itens "Lixo" e "Equipamentos públicos pichados", obtendo-se uma explicação de $86,2 \%$ da variância total e alfa de Cronbach de 0,820. Apesar da relativa pequena diferença numérica entre as medianas nos estratos de IVS, vizinhanças com baixa vulnerabilidade possuíam um indicador maior em relação àquelas com vulnerabilidade elevada $(\mathrm{p}=0,001)$, indicando que as distribuições dos escores de D6 são diferentes entre os estratos.

Na composição do domínio D7 permaneceram apenas três itens ("Iluminação pública”, "Itens de segurança" e "Policiamento"), com 79,7\% de explicação da variância total e alfa de Cronbach igual a 0,751 . Vizinhanças com IVS baixo apresentam indicadores com medianas maiores, diminuindo na direção da elevação do IVS ( $\mathrm{p}<0,001)$.

O domínio D8 foi composto por três subdomínios cujas medianas foram significativamente diferentes quando considerados os diversos estratos de IVS. O primeiro subdomínio ficou representado pelos itens "Local de compra de alimentos para serem preparados", "Local para lanches rápidos", "Local para compra de verduras", "Saúde privado" e "Academia pública ou privada”; o segundo, por "Coletor de lixo público ou privado" e "Ensino fundamental e médio"; e o terceiro, representado apenas pela presença de estabelecimento de serviços de mecânicos ou acessórios automotivos. O alfa de Cronbach foi de 0,674 e, juntos, os três subdomínios explicaram 74,5\% da variância total.

É importante salientar que todos os indicadores apresentados foram avaliados estatisticamente, considerando o teste não paramétrico de Kruskal-Wallis, que considera a posição dos postos das observações amostrais. Nesse teste, as médias dos postos de cada grupo (estrato) são comparadas, e não as medianas das observações. Porém, com o objetivo de facilitar a interpretação e padronizar a apresentação dos resultados, os dados de cada estrato de IVS foram representados pela mediana e distância interquartílica. Assim, é possível obter-se uma significância estatística, mesmo quando as medianas dos grupos são numericamente similares, já que o teste de Kruskal-Wallis é mais amplo e avalia se a distribuição de valores amostrais é similar entre os vários grupos.

\section{Discussão}

Os indicadores construídos evidenciaram heterogeneidade necessária em relação às vizinhanças frente aos diferentes riscos de vulnerabilidade social. $\mathrm{O}$ critério adotado ao avaliar os indicadores por meio de suas distribuições perante as diferentes categorias de IVS deu-se pelo fato de o IVS ser uma ferramenta de entendimento direto e com boa capacidade de discriminar as desigualdades entre 


\section{Figura 1}

Distribuição dos indicadores compostos e seus subdomínios, de acordo com a categoria do Índice de Vulnerabilidade da Saúde (IVS).

1a) Condições das ruas e itens de trânsito

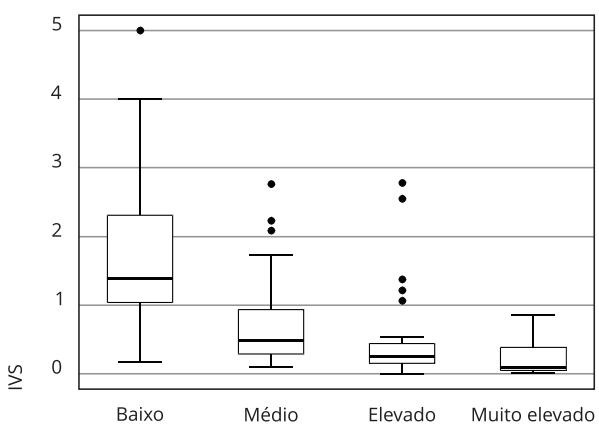

1c) Local para prática de atividade física e lazer

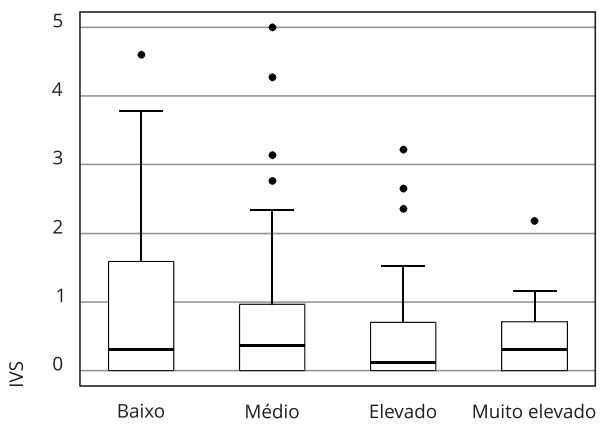

1e) Estético

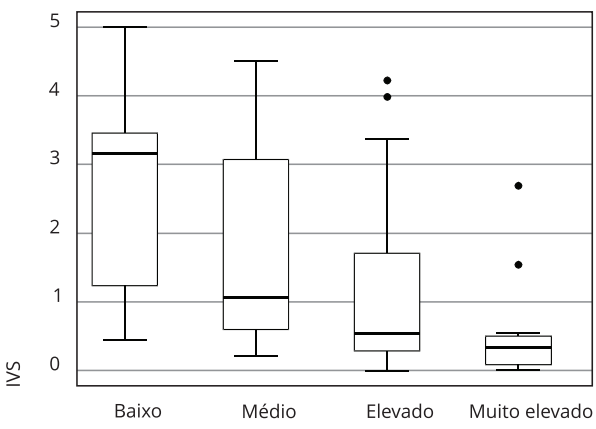

1g) Segurança

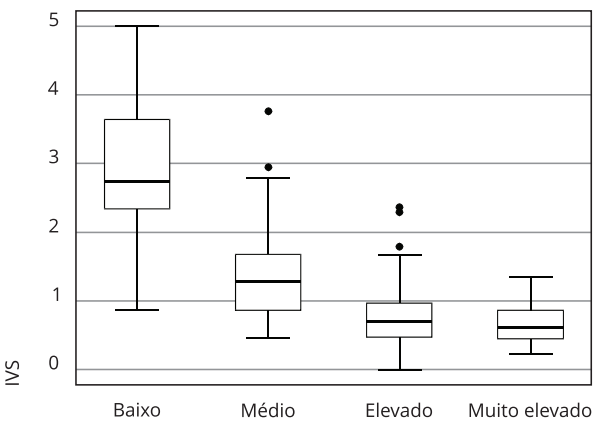

1b) Mobilidade

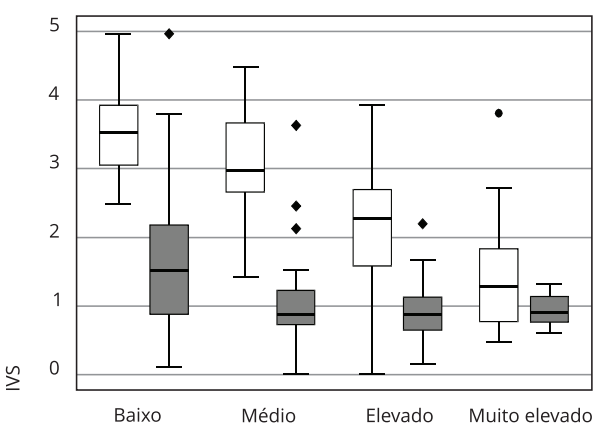

| Subdomínio 2.1

Subdomínio 2.2

1d) Caracterização dos imóveis

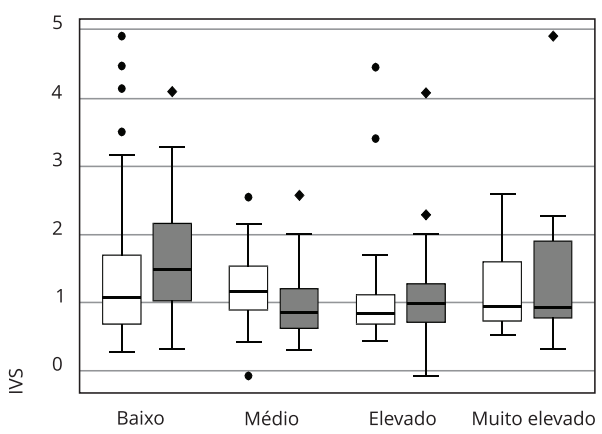

Subdomínio 4.1

Subdomínio 4.2

1f) Desordem física

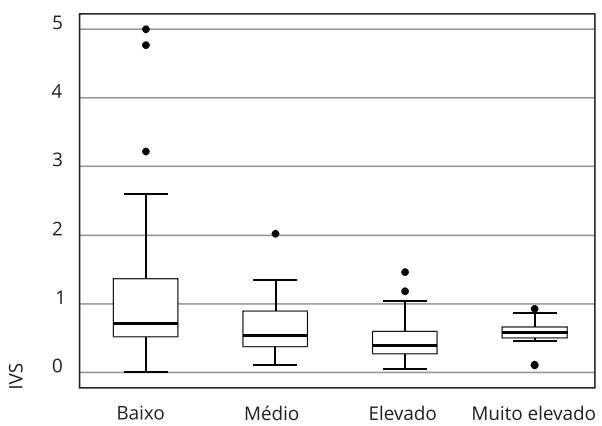

1h) Serviços

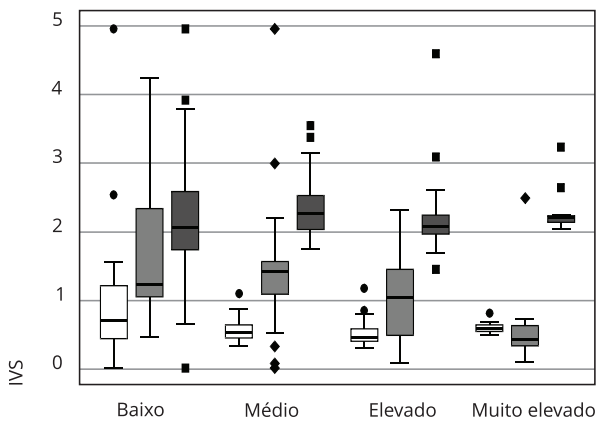

7 Subdomínio 8.1

Subdomínio 8.2

Subdomínio 8.3 
os diferentes territórios da cidade, além de ser amplamente usado para nortear políticas públicas de saúde em Belo Horizonte 33 .

Os domínios propostos apresentaram consistência interna aceitáveis, com alfa de Cronbach variando de 0,558 a 0,820. Os atributos físicos e sociais avaliados mostraram-se coerentes com a distribuição do IVS. Consideremos, por exemplo, o domínio D1 (Condições das ruas e itens de trânsito). Apesar de ser formado por apenas três itens, eles captaram, de forma coerente, a diferença entre as categorias de IVS. Vizinhanças com maior vulnerabilidade social apresentaram maior proporção de segmentos do tipo beco. Como consequência, tal tipo de segmento caracterizou-se por menor fluxo de veículos e escassa presença de itens de trânsito e sinalização.

A técnica estatística de análise de componentes principais conseguiu discernir bem os itens avaliados com relação à mobilidade (domínio D2), gerando dois subdomínios com características bastante próprias. O primeiro foi constituído de atributos que, de certa forma, tornam mais agradável o deslocamento dos pedestres, como passeios pavimentados, sombras projetadas por árvores, largura adequada do passeio e percepção do observador de ambiente agradável para deslocamento. Essas características estiveram mais presentes em vizinhanças com baixa vulnerabilidade, o que fortalece a prerrogativa de que o ambiente com boa estrutura física favorece o deslocamento e cria oportunidades da prática de atividade física para indivíduos que vivem no entorno dessas áreas 35 . O segundo subdomínio conseguiu captar a presença de itens que facilitam o deslocamento, como rampa de acesso e piso tátil, e proporcionam mais segurança, como presença de grades que delimitam o fluxo de veículos, faixas de pedestres e passarelas. Novamente, esses itens foram mais observados em vizinhanças com IVS baixo, o que pode demonstrar que áreas com piores estratos de IVS necessitam de melhores condições no contexto de suas vizinhanças 36 .

Porém, vale ressaltar que, em todas as vizinhanças analisadas, os atributos que facilitavam a prática de atividade física e socialização tiveram baixas proporções e, via de regra, distribuíram-se de forma homogênea em relação aos estratos de IVS. Esse resultado ressalta a importância para o redirecionamento e a valorização de políticas de saúde que incentivam a prática de atividade física e acesso a equipamentos e áreas de lazer 37.

Em relação à caracterização dos imóveis (domínio D4), apesar do indicador composto apresentar pouca heterogeneidade, os indicadores simples possibilitaram verificar maior presença de imóveis em construção/reforma e propaganda de venda de imóveis nas vizinhanças de IVS baixo, provavelmente indicando uma atividade imobiliária da cidade em áreas com menor vulnerabilidade e, consequentemente, melhores condições 38 .

Um comportamento semelhante pode ser observado em relação aos atributos relacionados à estética do ambiente (domínio D5). Vizinhanças mais favorecidas apresentaram uma presença maior de jardins e árvores e ambientes limpos, enquanto aquelas menos favorecidas tiveram maior presença de propaganda de eventos políticos e música, corroborando os achados dos estudos que sugerem maior coesão social nessas vizinhanças ${ }^{39}$. Também foi possível observar a maior presença de imóveis pichados e/ou deteriorados (domínio D6), coerente com achados de outros autores 40,41. Tais áreas, com maiores indicadores de desordem física, apresentaram um baixo indicador referente a atributos de segurança (domínio D7). Quando avaliados os indicadores simples para esse domínio, percebemos claramente a discrepância entre os estratos de IVS.

Em relação aos itens de serviços (domínio D8), apesar dos indicadores compostos se apresentarem mais homogêneos em relação aos estratos de IVS, é possível observar que atributos referentes ao acesso a alimentos e a saúde privada são mais presentes em vizinhanças com menor risco de vulnerabilidade. Coletores de lixo público ou privado e escolas de ensino fundamental e médio também têm menor presença nos estratos de IVS muito elevado.

Algumas limitações deste estudo devem ser mencionadas. A mensuração de alguns atributos pode ser limitada, pois certos itens são passíveis de variação temporal. Uma medição mais confiável exigiria mais de uma observação para o mesmo segmento, de forma que horários e dias diferentes fossem considerados. É preciso ter um objeto bem definido e conhecimento prévio dos aspectos relevantes para a pesquisa. Por ser um instrumento relativamente fácil e rápido, o pesquisador pode se ver tentado a coletar dados em excesso, sobrecarregando o observador e aumentando as chances de erros na coleta.

Entretanto, vale também evidenciar várias vantagens da OSS. O instrumento permite atuar com outras técnicas de investigação, sendo facilmente adaptável quanto à metodologia e ao objeto de 
estudo. Pode ser utilizado em diferentes formas, como questionários, gravações de vídeos, tablets ou até ferramentas como Google Street View (https://www.google.com/streetview). É possível fazer o levantamento de dados quantitativos e qualitativos em uma mesma investigação e reprodução da ferramenta para uso dos demais pesquisadores, mostrando-se interessante para a sua difusão e aperfeiçoamento.

Os resultados deste estudo podem contribuir para estudos futuros, como analisar os indicadores com variáveis individuais e de contexto, possibilitando estudos multiníveis para melhor compreender a saúde das populações aninhadas na vizinhança estudada. Possibilitarão, ainda, investigar como os indicadores se comportarão diante de diferentes limites de vizinhança e em áreas urbanas de cidades com diferentes populações, ocupações territoriais e histórias, principalmente sob a observação sistemática de um observador, evitando a possível imprecisão do caráter subjetivo da informação e o viés de "fonte comum", plausível dos inquéritos populacionais.

\section{Colaboradores}

D. A. S. Costa e S. A. Mingoti participaram da análise e da interpretação dos dados, da elaboração do manuscrito, da revisão crítica relevante do conteúdo e da aprovação da versão final do manuscrito. A. C. S. Andrade participou da análise e da interpretação dos dados, da elaboração do artigo e da aprovação da versão final do manuscrito. C. C. Xavier e F. A.o Proietti participaram da concepção e do planejamento do projeto. W. T. Caiaffa participou da concepção e do planejamento do projeto, da interpretação dos dados, da elaboração do manuscrito, da revisão crítica relevante do conteúdo e da aprovação da versão final do manuscrito.

\section{Agradecimentos}

A todos os pesquisadores do Observatório de Saúde Urbana de Belo Horizonte que participaram do Estudo Saúde em Beagá, ao apoio da Secretaria Municipal de Saúde de Belo Horizonte na coleta de dados e ao Conselho Nacional de Desenvolvimento Científico e Tecnológico (CNPq) pela bolsa produtividade em pesquisa da autora W. T. Caiaffa. Ao Fundo Nacional de Saúde do Ministério da Saúde, Fundação de Amparo à Pesquisa do Estado de Minas Gerais (Fapemig), CNPq e NIH/Fogarty International Center pelo financiamento. 


\section{Referências}

1. Diez Roux AV. Bringing context back into epidemiology: variables and fallacies in multilevel analysis. Am J Public Health 1998; 88:216-22.

2. Minayo MCS. Saúde e ambiente: uma relação necessária. In: Campos GWS, Minayo MCS, Akerman M, Júnior MD, Carvalho YM, organizadores. Tratado de saúde coletiva. São Paulo: Editora Hucitec; 2006. p. 93-122.

3. Dunn JR, Cummins S. Placing health in context. Soc Sci Med 2007; 65:1821-4

4. Barcellos C, Monken M. O território na promoção e vigilância em saúde. In: Fonseca $\mathrm{AF}$ organizador. O território e o processo saúdedoença. Rio de Janeiro: Escola Politécnica de Saúde Joaquim Venâncio, Fundação Oswaldo Cruz; 2007. p. 177-224.

5. Diez Roux A, Evenson KR, McGinn AP, Brown DG, Moore L, Brines S, et al. Availability of recreational resources and physical activity in adults. Am J Public Health 2007; 97:493-9.

6. Reiss Jr. AJ. Systematic observation of natural social phenomena. In: Costner HL, editor. Sociological methodology. San Francisco: JosseyBass, Inc.; 1971. p. 3-33.

7. Rosenshine B, Furst NF. The use of direct observation to study teaching. In: Gage NL, editor. Handbook of research on teaching. Chicago: Rand-McNally; 1973. p. 122-83.

8. Mastrofski SD, Parks RB, Reiss Jr. AJ, Worden RE, DeJong C, Snipes JB, et al. Systematic observation of public police: applying field research methods to policy. Washington DC: National Institute of Justice; 1998.

9. Proietti FA, Oliveira CDL, Ferreira FR, Ferreira AD, Caiaffa WT. Unidade de contexto e observação social sistemática em saúde: conceitos e métodos. Physis (Rio J.) 2008; 18:469-82

10. Diez Roux AV. Residential environments and cardiovascular risk. J Urban Health 2003; 80:569-89.

11. Cubbin C. Protective and harmful effects of neighborhood-level deprivation on individuallevel health knowledge, behavior changes, and risk of coronary heart disease. Am J Epidemiol 2005; 162:559-68.

12. Li F, Harmer P, Cardinal BJ, Bosworth M, Johnson-Shelton D, Moore JM, et al. Built environment and 1-year change in weight and waist circumference in middle-aged and older adults: Portland Neighborhood Environment and Health Study. Am J Epidemiol 2008; 169:401-8

13. Lima-Costa MFF. A saúde dos adultos na Região Metropolitana de Belo Horizonte: um estudo epidemiológico de base populacional. Belo Horizonte: Núcleo de Estudos em Saúde Pública e Envelhecimento; 2004.
14. Friche AA, Diez Roux AV, César CC, Xavier CC, Proietti FA, Caiaffa WT. Assessing the psychometric and ecometric properties of neighborhood scales in developing countries: Saúde em Beagá Study, Belo Horizonte, Brazil, 2008-2009. J Urban Health 2013; 90:246-61.

15. Andrade ACS, Peixoto SV, Friche AAL, Goston JL, César CC, Xavier CC, et al. Social context of neighborhood and socioeconomic status on leisure-time physical activity in a Brazilian urban center: The BH Health Study. Cad Saúde Pública 2015; 31 Suppl:S136-47.

16. Raudenbush SW, Sampson RJ. Ecometrics: toward a science of assessing ecological settings, with application to the systematic social observation of neighborhoods. Sociol Methodol 1999; 29:1-41.

17. Suminski RR, Heinrich KM, Poston WSC, Hyder M, Pyle S. Characteristics of urban sidewalks/streets and objectively measured physical activity. J Urban Health 2007; 85:178-90.

18. Cohen D, Spear S, Scribner R, Kissinger P, Mason K, Wildgen J. "Broken windows" and the risk of gonorrhea. Am J Public Health 2000; 90:230-6.

19. Saavedra DA. Autoavaliação do estado de saúde e qualidade da vizinhança dos moradores da área central do município de São Paulo [Dissertação de Mestrado]. São Paulo: Faculdade de Ciências Médicas da Santa Casa de São Paulo; 2011

20. Oliveira CDL, Diez Roux AV, César CC, Proietti FA. A case-control study of microenvironmental risk factors for urban visceral leishmaniasis in a large city in Brazil, $1999-2000$ Rev Panam Salud Pública 2006; 20:369-76.

21. Friche AAL, Xavier CC, Proietti FA, Caiaffa WT, organizadores. Saúde urbana em Belo Horizonte. Belo Horizonte: Editora UFMG; 2015

22. Caiaffa WT, Ferreira FR, Ferreira AD, Oliveira CLO, Camargos VP, Proietti FA. Saúde urbana: "a cidade é uma estranha senhora, que hoje sorri e amanhã te devora”. Ciênc Saúde Coletiva $2008 ; 13: 1785-96$.

23. Freitas ED, Camargos VP, Xavier CC, Caiaffa WT, Proietti FA. Instrumento para condução de observação social sistemática: métodos e resultados da concordância interobservadores. Cad Saúde Pública 2013; 29:2093-104.

24. Instituto Brasileiro de Geografia e Estatística. Tendências demográficas: uma análise dos resultados da sinopse preliminar do Censo Demográfico 2000. Rio de Janeiro: Instituto Brasileiro de Geografia e Estatística; 2001. 
25 Ferreira AD, César CC, Malta DB, Andrade ACS, Ramos CGC, Proietti FA, et al. Validade de estimativas obtidas por inquérito telefônico: comparação entre VIGITEL 2008 e inquérito Saúde em Beagá. Rev Bras Epidemiol 2011; 14:16-30.

26. Célio FA, Xavier CC, Andrade ACS, Camargos VP, Caiaffa WT, Friche AAL, et al. Características individuais associadas à autopercepção da extensão territorial da vizinhança. Cad Saúde Pública 2014; 30:1935-46.

27. Chaskin R. Perspectives on neighborhood and community: a review of the literature. Soc Serv Rev 1997; 71:521-47.

28. Freitas ED. Observação Social Sistemática: aplicação da observação direta das condições físicas e sociais de uma vizinhança [Dissertação de Mestrado]. Belo Horizonte: Programa de Pós-graduação em Saúde Pública, Universidade Federal de Minas Gerais; 2013.

29. Bolfarine H, Bussab WO. Elementos de amostragem. São Paulo: Editora Blucher; 2005.

30. Mingoti SA. Análise de dados através de métodos de estatística multivariada: uma abordagem aplica. Belo Horizonte: Editora UFMG; 2005.

31. Hair JF, Black WC, Babin BJ, Anderson RE, Tatham RL. Análise multivariada de dados. 6a Ed. Porto Alegre: Bookman; 2009.

32. Instituto Brasileiro de Geografia e Estatística. Censo Demográfico 2010. Características da população e dos domicílios: resultados do universo. Rio de Janeiro: Instituto Brasileiro de Geografia e Estatística; 2011.

33. Gerência de Epidemiologia e Informação, Secretaria Municipal de Saúde de Belo Horizonte. Índice de vulnerabilidade à saúde, 2012. http://portalpbh.pbh.gov.br/pbh/ecp/files. do? evento=download\&urlArqPlc =Ivsaude-ris co2012.pdf.

34. Siegel S, Castellan Jr. NJ. Estatística não paramétrica para as ciências do comportamento. São Paulo: Artmed Bookman; 2006.
35. Hino AA, Reis RS, Sarmiento OL, Parra DC, Brownson RC. The built environment and recreational physical activity among adults in $\mathrm{Cu}-$ ritiba, Brazil. Prev Med 2011; 52:419-22.

36. World Health Organization. Promoting physical activity and active living in urban environments. Geneva: World Health Organization; 2006.

37. World Health Organization. Global recommendations on physical activity for health. Geneva: World Health Organization; 2011.

38. Fingerhut L, Lovasi G. Supporting evidencebased investment in public spaces: a literature review. The Haven Project. New York: Mailman School of Public Health, Columbia University; 2015.

39. Lovasi GS, Jacobson JS, Quinn JW, Neckerman $\mathrm{KM}$, Ashby-Thompson MN, Rundle A. Is the environment near home and school associated with physical activity and adiposity of urban preschool children? J Urban Health 2011; 88:1143-57.

40. Lovasi GS, O'Neil-Dunne JP, Lu JW, Sheehan D, Perzanowski MS, MacFaden SW, et al. Urban tree canopy and asthma, wheeze, rhinitis, and allergic sensitization to tree pollen in a New York City birth cohort. Environ Health Perspect 2013; 121:494-500.

41. Foster S, Giles-Corti B, Knuiman M. Creating safe walkable streetscapes: does house design and upkeep discourage incivilities in suburban neighbourhoods? J Environ Psychol 2011; 31:79-88. 


\section{Abstract}

Place of residence is heavily shaped by social position, indicating that neighborhood characteristics can be important contributing factors to health iniquities. The objectives were to construct indicators of the physical and social environment in an urban context based on variables obtained with the Systematic Social Observation method (SSO) and to analyze them according to the Health Vulnerability Index (HVI). The instrument was developed to determine the characteristics of the physical and social neighborhood in two health districts in Belo Horizonte, Minas Gerais State, Brazil. Data were collected from April to June 2011. To develop the simple indicators, ratios were calculated for the number of observed items per residence in each segment. Composite indicators were built using principal components analysis via covariance matrix. The final sample consisted of 1,295 street segments nested in 147 neighborhoods. Indicators referring to street conditions and transit items, mobility, appearance, housing and property, physical disorder, safety/security, and services showed a dose-response behavior in relation to HVI ( $p<$ 0.05). Indicators pertaining to place to practice physical activity and leisure did not show significant differences. The indicators displayed coherent behavior towards different HVI strata and proved adequate within each respective domain and subdomain.

Residence Characteristics; Social Indicators; Urban Health

\section{Resumen}

El lugar de residencia está fuertemente modelado por la posición social, indicando qué características del vecindario pueden contribuir de forma importante a las inequidades en salud. El objetivo de este estudio fue construir indicadores del ambiente físico y social en un contexto urbano, a partir de las variables obtenidas por el método de Observación Social Sistemática (OSS) y analizarlos de acuerdo con el Índice de Vulnerabilidad de la Salud (IVS). El instrumento se desarrolló con el objetivo de conocer las características del entorno físico y social del lugar de residencia de los habitantes de dos distritos sanitarios de Belo Horizonte, Minas Gerais, Brasil. Los datos se recogieron entre los meses de abril y junio de 2011. En la construcción de los indicadores simples, se calcularon las ratios del número de items observados por residencia para cada segmento. En la construcción de los indicadores compuestos, se utilizó el método del análisis de componentes principales vía matriz de covariancias. La muestra final estuvo compuesta por 1.295 segmentos de calles anidados en $147 \mathrm{ve}$ cindarios. Percibimos que los indicadores, referentes a las condiciones de las calles y elementos de tránsito, movilidad, estético, caracterización de los inmuebles, desorden físico, seguridad y servicios presentaron un comportamiento dosis-respuesta en relación con el IVS (valor de $p<0,05)$. Los indicadores concernientes al lugar para la práctica de la actividad física y ocio no presentaron diferencias significativas. Los indicadores demostraron un comportamiento coherente ante diferentes estratos del indice de vulnerabilidad de la salud y se mostraron adecuados dentro de cada dominio y subdominio creados.

Distribución Espacial de la Población; Indicadores Sociales; Salud Urbana
Recebido em 16/Fev/2016

Versão final reapresentada em 18/Ago/2016 Aprovado em 14/Out/2016 